\title{
The Chemistry of CRL 618
}

\author{
Paul M. Woods, T.J. Millar, Albert A. Zijlstra \\ Department of Physics, UMIST, P.O. Box 88, Manchester, M60 1QD, \\ $U K$ \\ E. Herbst \\ Departments of Physics and Astronomy, The Ohio State University, \\ Columbus, $\mathrm{OH}$ 43210, USA.
}

\begin{abstract}
We present a chemical model of the central torus around CRL 618 in order to confirm that long-chained hydrocarbons and benzene are formed at detectable abundances. We reproduce the observed abundances of these molecules comparatively well. We infer from our calculations that CRL 618 must be a young PPN, with a post-AGB age of approximately $600 \mathrm{yr}$.
\end{abstract}

The discoveries of previously undetected hydrocarbon species made by ISO in the proto-planetary nebula (PPN) CRL 618 have shown that some interesting chemistry occurs during the transition from AGB stars to $\mathrm{PNe}$. Cernicharo and collaborators report detections of the hydrocarbons $\mathrm{C}_{4} \mathrm{H}_{2}, \mathrm{C}_{6} \mathrm{H}_{2}, \mathrm{C}_{2} \mathrm{H}_{4}, \mathrm{CH}_{3} \mathrm{C}_{2} \mathrm{H}$ and $\mathrm{CH}_{3} \mathrm{C}_{4} \mathrm{H}$, in addition to the cyclic molecule, benzene $\left(c-\mathrm{C}_{6} \mathrm{H}_{6}\right.$. Cernicharo et al. 2001, A\&A, 546, L123; L127).

We present a chemical model which represents the inner, dusty torus of CRL 618. The model considers the chemistry of a dense, slowly expanding shell irradiated by a strong central UV field. It is in these conditions that we expect a rich photochemistry to develop, leading to the formation of complex molecules. We aim to reproduce the observed abundances of benzene, in particular, but also other large hydrocarbons.

Further details of the chemical model can be found in Millar, Herbst \& Bettens (2000, MNRAS, 316, 195). Physical parameters were adapted from Herpin \& Cernicharo (2000, ApJ, 530, L129), viz., $R_{i}=1.810^{15} \mathrm{~cm}, v_{\exp }=$ $1 \mathrm{kms}^{-1}, T=250 \mathrm{~K}, n_{0}\left(\mathrm{H}_{2}\right)=1.2510^{8} \mathrm{~cm}^{-3}, G=210^{5} G_{0}, \zeta=1.310^{-15} \mathrm{~s}^{-1}$. We have increased the cosmic ray ionisation rate $(\zeta)$ by a factor 100 over the interstellar value to account for some energetic ionisation process, e.g. X-ray ionisation. The shell has a constant temperature, and it expands with a constant velocity and a density which varies as $1 / r^{2}$.

Fractional abundances are calculated by solving the rate equations for 3878 reactions. Column densities are calculated by integrating along the line of sight, as a function of time. CO self-shielding is incorporated in the manner of Mamon et al. (1988, ApJ, 328, 797).

We reach fairly good agreement with observations. Abundances of small molecules (e.g. $\mathrm{C}_{2} \mathrm{H}, \mathrm{C}_{3} \mathrm{H}, \mathrm{C}_{4} \mathrm{H}$ ) in our model are noticeably low; this suggests that our model is too efficient at producing large molecules. We can improve 


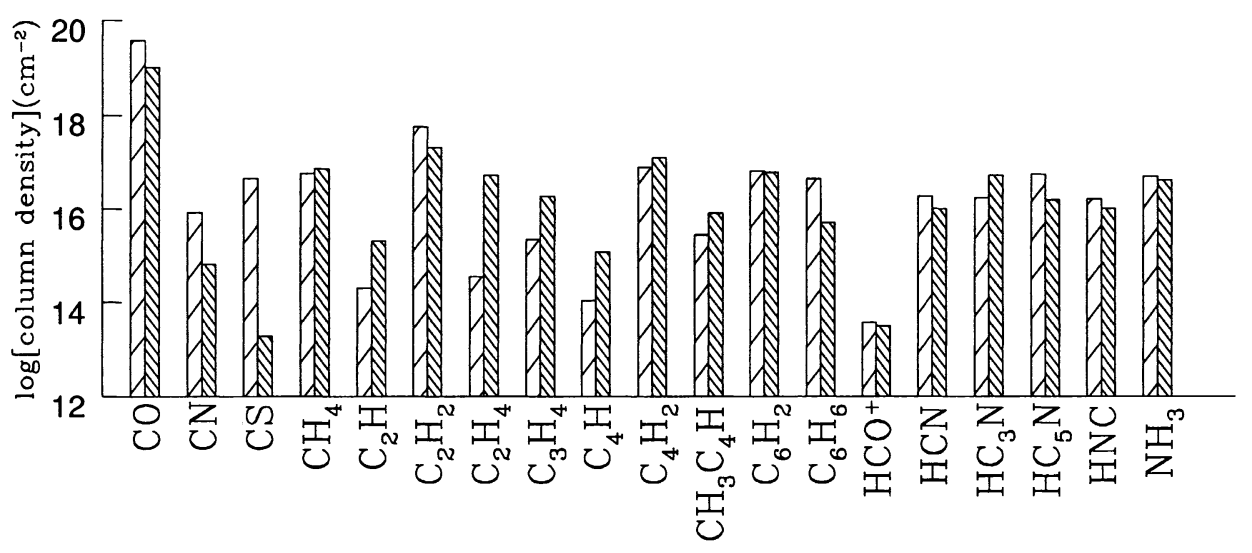

Figure 1. Observed (dense hatching) versus model column densities.

on this by incorporating more destruction mechanisms for longer molecules. Also low is our calculated abundance of $\mathrm{HCO}^{+}$. This is possibly due to the contribution shock chemistry makes to $\mathrm{HCO}^{+}$abundance in CRL 618. However, it may be that there are ionising X-rays emitted from CRL 618, although Cox et al. (1992, A\&A, 266, 420) do not favour this idea. The abundance of benzene reaches $10^{-6}$ molecules per $\mathrm{H}_{2}$ molecule, as does the abundance of the yetunobserved cyclic molecule, $c-\mathrm{C}_{6} \mathrm{H}_{5} \mathrm{CN}$. Similarly high abundances are seen in large carbon-chain molecules $\left(\mathrm{C}_{n}, \mathrm{C}_{n} \mathrm{H}, \mathrm{C}_{n} \mathrm{H}_{2}\right)$ for $n$ up to 13 . Peak abundances for the cyanopolyynes are $7.610^{-7}, 1.610^{-6}, 1.310^{-6}, 9.010^{-7}, 7.010^{-7}$ and $4.310^{-7}$ for $\mathrm{HC}_{3} \mathrm{~N}$ through to $\mathrm{HC}_{13} \mathrm{~N}$.

Figure 1 shows a comparison between calculated and observed column densities. Of the 19 species we have observed column densities for, we are within a factor 10 for 14 of these. Of the remaining 5, observed columns for CN and CS are questionably low (Bachiller et al. 1997, A\&A, 319, 235) and $\mathrm{C}_{2} \mathrm{H}, \mathrm{C}_{4} \mathrm{H}$ and $\mathrm{C}_{2} \mathrm{H}_{4}$ are amongst 'small' molecules previously mentioned.

We find that to obtain good agreements with between observed and calculated column densities and abundances, the lifetime of CRL 618 in the post-AGB phase must be approximately $600 \mathrm{yr}$. This is in agreement with the lifetime that the paucity of PPNe suggests. Reasonable results are obtained if the lifetime is assumed to be $200-800 \mathrm{yr}$. The calculated age relies heavily on the assumed density and expansion velocity; however too low a density or too high a velocity means abundances much lower than those observed, and an age which is not realistic physically.

The chemistry of CRL 618 is very rich and warrants further study. High abundances of complex species are seen, and these can be reproduced with a chemical model. From this model, the age of CRL 618 as a post-AGB object has been determined to be approximately $600 \mathrm{yr}$. 